\title{
真实有雾场景下的目标检测
}

\author{
解宇虹 ${ }^{1)}$ ，谢源2)，陈亮1)，李翠华1)，曲延云1)* \\ ${ }^{1)}$ (厦门大学信息学院 厦门 361000) \\ 2) (华东师范大学计算机科学与技术学院 上海 200062) \\ (yyqu@xmu.edu.cn)
}

\begin{abstract}
摘 要: 在有雾场景中实现对目标精确检测，是视频监控、智慧城市、无人驾驶等多个实际应用中一个重要的研究 内容. 为促进真实有雾场景下的目标检测研究, 探讨了 2 个问题: 有雾场景目标检测数据集的构建以及真实有雾场景 下目标检测的解决方案. 首先, 设计了一种系统化的、具有真实感的有雾图像合成方法, 并建立了合成有雾场景的目 标检测数据集. 同时, 探讨了对真实有雾场景下目标检测器具有提升性能作用的数据集处理方法. 其次, 探讨了先验 知识和模型的联合优化对真实有雾场景的目标检测性能的有效性，并提出了 2 个框架：基于知识引导的目标检测框架 和基于图像去雾和目标检测的联合学习框架. 基于知识引导的目标检测框架将统计先验知识用于指导通用目标检测 网络学习有雾场景下的目标特征, 使通用目标检测器能更好地适应特殊的目标检测场景. 基于图像去雾和目标检测 的联合学习框架通过去雾模型和目标检测模型的联合优化学习, 有效学习图像去雾中恢复的结构细节和颜色特征, 从而提高真实有雾场景下的目标检测精度. 在 RTTS 数据集上的实验结果表明，基于知识引导的目标检测框架和基于 图像去雾和目标检测的联合学习框架能够有效地提高有雾场景下目标检测器的性能, 均值平均精度(mAP)分别为 $70.5 \%$ 和 $66.6 \%$
\end{abstract}

关键词：真实有雾场景; 目标检测; 图像去雾; 知识引导; 联合学习

中图法分类号: TP391.41 DOI: 10.3724/SP.J.1089.2021.18554

\section{Object Detection in Real-World Hazy Scene}

\author{
Xie Yuhong ${ }^{1)}$, Xie Yuan ${ }^{2)}$, Chen Liang $^{1)}$, Li Cuihua ${ }^{1)}$, and Qu Yanyun ${ }^{1{ }^{*}}$ \\ 1) (School of Information, Xiamen University, Xiamen 361000) \\ 2) (School of Computer Science and Technology, East China Normal University, Shanghai 200062)
}

\begin{abstract}
Accurate object detection in the real-world hazy scene is very important to some potential visual task, such as video surveillance, smart city, autonomous driving and so on. This paper focuses on two research problems, which are to build a synthetic dataset of object detection in hazy scene and to analyze the effect of prior knowledge and joint learning of model on object detection in real-world hazy scene. Two frameworks are proposed which are the knowledge-guided object detection (KODNet) and the joint learning in dehazing and object detection (DONet). In KODNet, statistical prior knowledge will be used to guide the general object detection network to learn object features in the hazy scene during the training, makes the general object detector better adapt to the special object detection scenario. DONet can effectively solve the problem of structural detail missing and color distortion caused by image dehazing, thereby realizing the improvement of the objects
\end{abstract}

收稿日期：2020-08-05; 修回日期：2020-11-18. 基金项目：国家自然科学基金面上项目(61876161，61772524); 海峡联合基金 (U1065252); 北京市自然科学基金面上项目(4182067). 解宇虹(1994-), 女, 硕士研究生, 主要研究方向为计算机视觉、深度学习; 谢源(1985-), 男, 博士, 教授, 博士生导师, 主要研究方向为图像处理、计算机视觉、机器学习和模式识别; 陈亮(1996-), 男, 硕士 研究生, 主要研究方向为计算机视觉、深度学习; 李翠华(1960-), 男, 博士, 教授, 博士生导师, CCF 会员, 主要研究方向为计算机 视觉、视频与图像处理、超分辨率重建; 曲延云(1972-), 女, 博士, 教授, 博士生导师, CCF 会员, 论文通讯作者, 主要研究方向为 图像处理、计算机视觉、机器学习和模式识别. 
detection accuracy in real-world scene. The experimental results on RTTS show that KODNet and DONet are effective to the object detection in the real-world hazy scene and they achieve the mAP of $70.5 \%$ and $66.6 \%$.

Key words: real-world hazy scene; object detection; image dehazing; knowledge-guided method; joint learning

目标检测一直是计算机视觉领域中一个热点 研究方向, 特别是随着深度学习的兴起, 基于深度 学习的目标检测方法取得了突破性进展. 然而, 现 有的目标检测研究大多是针对简单干净环境下的 目标，这样的目标检测模型，虽然在已有的目标检 测数据集上取得好的检测结果，但是真正应用于 真实世界有雾的环境中时, 往往效果不佳. 真实世 界中的有雾场景往往是无限制的室外开放环境, 因此该环境下拍摄的图像的降质因素是动态变化 的. 以雾天城市街景为例, 所处的场景是存在动态 目标、烟尘悬浮物和汽车尾气等不确定因素的环 境. 这些因素使拍摄的图像受到模糊、遮挡、光照 异常等影响, 造成图像细节缺失、对比度低和颜色 失真等问题. 随着人工智能时代的到来, 对能落地 的智能算法需求日益迫切，真实有雾场景下的目 标检测涉及视频监控、智慧城市、无人驾驶及军事 应用，成为当前亟待解决的问题，引起了相关研 究人员广泛的关注.

2017 年, $\mathrm{Li}$ 等 ${ }^{[1]}$ 提出了第 1 个端到端的去雾网 络 AODNet, 并且讨论了图像去雾对目标检测任务 的影响. 其选取目标检测网络 Faster R-CNN ${ }^{[2]}$ 与 AODNet 进行联合优化训练, 并进行目标检测的评 估，这是第 1 个涉及合成有雾场景下目标检测的研 究. 此后该课题组针对真实有雾场景下的目标检 测做了相关的研究 ${ }^{[3-5]}$. 他们首先发布大型的合成 有雾图像数据集 $\mathrm{RESIDE}^{[3]}$ ，该数据集包括真实 世界有雾场景下的目标检测数据集(annotated realworld task-driven testing set, RTTS), 这是目前唯一 带多类检测标签的真实有雾场景的目标检测数据 集. 接着，该课题组对图像去雾任务以及有雾图像 的目标检测任务进行深人研究, 并提出了对图像 先去雾再使用原始目标检测器对去雾后图像进行 检测的方法(后文统一称之为基于去雾-检测的无 关联目标检测方法)和对图像先去雾再使用自适应 目标检测器对去雾后图像进行检测的方法，从而 解决真实有雾场景下的目标检测问题. 2019 年，在 举办 IEEE CVPR2019 UG ${ }^{2}+$ 挑战赛中, Yang 等 ${ }^{[5]}$ 发 布了真实有雾场景下目标检测的基准，希望有更 多学者参与真实有雾场景下的目标检测研究.
虽然已有针对真实有雾场景下的目标检测的 研究, 但仍存在大量的问题亟待解决. 本文主要研 究 2 个问题. (1) 数据集缺乏. 现有的数据集要么 是用于目标检测的，如 PASCAL VOC ${ }^{[6]}$ 和 MS $\mathrm{COCO}^{[7]}$, 要么是单纯用于图像去雾的, 如 $\operatorname{HSTS}^{[3]}$, 其在真实有雾场景下的带目标标记的数据集非常 少. 目前包含多个类别的且公开的真实有雾场景 下目标检测数据集仅有 RESIDE RTTS 数据集. 而 深度模型对数据的需求极大, 没有大量的训练数 据, 就难以得到鲁棒且效果不错的深度模型。(2) 解决方案单一. 目前有 2 类方法可用于有雾场景下 的目标检测: 一类方法是直接使用现有的通用目 标检测方法, 但检测器性能因图像细节缺失、对比 度低、颜色失真和目标分布不同等问题而大大降 低; 另一类方法是对图像先去雾再进行目标检测. 众所周知, 图像去雾技术已取得长足的进展, 出现 了一些效果显著的去雾方法, 如 DehazeNet ${ }^{[8]}$ 和 PFFNet $^{[9]}$, 去雾后可以使图像的视觉效果得到提 升. 但是将现有的深度目标检测模型应用于去雾 后的图像, 是否能够完美解决有雾场景下的目标 检测问题，以及如何使用正确的图像去雾方法以 辅助下游的目标检测任务, 这些问题都需要一一 探讨.

基于当前的 2 个难点，本文主要探讨了 2 个促 进真实有雾场景下目标检测研究的方向. 本文首 先进行有雾场景下目标检测数据集的构建和处理. 数据集是视觉研究任务的起点, 有了数据集, 才能 进行模型和方法的讨论. 然而, 有雾场景图像的收 集是一个非常费时耗力的任务, 并且有雾图像的 目标标记成本非常高. 考虑到当前已有较完备的 目标检测数据集，本文在目标检测数据集上合成 有雾场景下的目标检测数据集. 由于 MS COCO 是 一个典型的、具有完备目标类别标记信息的目标检 测数据集, 因此本文针对 MS COCO 设计了一套系 统化的合成有雾图像方法. 首先, 对每幅图像进行 深度估计并生成深度图像. 接着, 采用导向滤波 ${ }^{[10]}$ 方法对深度图像进行平滑, 生成具有真实感的深 度图像. 最后, 采用大气散射模型, 将滤波后的深 度图像和清晰图像合成有雾图像。最终建立了有 
雾场景下的目标检测数据集 (synthetic Microsoft common objects in context, S-COCO). 此外, 真实 有雾场景下的目标检测数据集具有采集难、标注难 的特点, 因此数据集中存在漏标、错标等噪声, 造 成有雾场景下目标检测性能的下降. 针对该问题 探讨了数据集处理方法对真实有雾场景下目标检 测性能是否有促进作用. 为进一步提升检测性能, 探讨了知识引导和模型联合优化对真实有雾场景 下目标检测的促进作用. 据此, 本文提出了基于知 识引导的目标检测框架 (knowledge-guided object detection，KODNet)和基于图像去雾和目标检测的 联合学习框架(joint learning in dehazing and object detection, DONet). KODNet 通过统计先验学习, 设 计了深度检测模型中针框面比, 以此指导真实有 雾场景下的目标检测. DONet 将去雾模型与目标检 测模型级联并进行联合学习，使网络同时完成图 像去雾任务和目标检测任务.

\section{1 相关工作}

\section{1 有雾场景目标检测数据集}

目前，真实有雾场景下的带目标标注的数据集 非常少，仅有 3 个. 第 1 个是 UFDD(unconstrained face detection dataset $)^{[11]}$, 它包含 6 种恶劣环境下 的人脸，其中包括有雾场景下的人脸，但该数据集 不适用于本文所研究的多个类别的检测场景. 第 2 个是 RESIDE RTTS 数据集, 它是一个公开的数据 集. 该数据集从网上收集了 4322 幅真实世界的有 雾图像, 其中包括大量交通和驾驶摄像头拍摄到 的图像. RTTS 使用与 VOC2007 相同的格式标注 5 类目标, 分别是汽车、自行车、摩托车、人和公共 汽车. 此外, 在 IEEE CVPR 2019 中的 $\mathrm{UG}^{2}+$ 挑战赛 中，组委会另外收集了一个 2987 幅真实有雾场景 的目标检测数据集作为测试集. 在本文中称这个 数据集为 UG-test 数据集. 该数据集同样使用 VOC2007 的格式标注与 RTTS 相同的 5 类目标，但 该测试集仅公布其中的 100 幅图像标签. 因此, 本 文研究针对 RTTS 数据集以及 UG-test 数据集中的 其中 100 幅图像进行测试. 同时, 为了训练稳定鲁 棒的有雾场景目标检测模型，提出了一种系统化 的、具有真实感的有雾图像合成方法, 将生成的数 据集用于该模型的训练.

\section{2 通用目标检测方法}

随着深度学习的崛起, 通用目标检测任务已 经取得突破性的成绩. 目前, 使用深度学习的通用
目标检测框架可以分为单阶段和双阶段 2 类.

\subsection{1 单阶段目标检测方法}

单阶段目标检测方法首先通过特征提取网络 进行特征提取, 接着使用一些特定的算法, 如设置 一定宽高比和尺度的针框, 对特征图中的每个特 征产生相应的预测框并对预测框进行分类回归, 其网络框架如图 1 所示. 该类方法包含 Overfeat ${ }^{[12]}$, $\mathrm{YOLO}^{[13-15]}, \mathrm{SSD}^{[16]}$, RetinaNet ${ }^{[17]}$, RefineDet ${ }^{[18]}$ 和 $\mathrm{FCOS}^{[19]}$ 等方法. 单阶段目标检测方法更注重网络 的检测速度，但精度会稍低于某些双阶段的目标 检测网络.

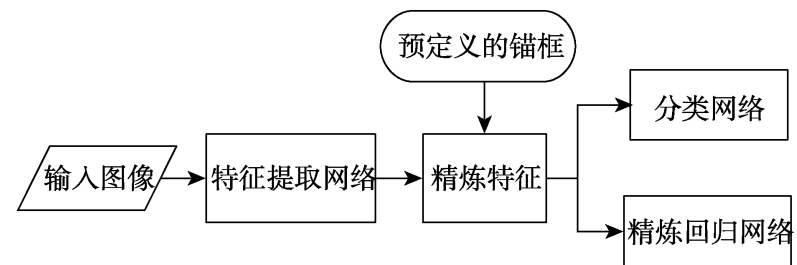

图 1 单阶段目标检测网络框架

\subsection{2 双阶段目标检测方法}

双阶段目标检测方法一般是通过区域生成网 络(region proposal network, RPN)先产生一定的候 选区域，再对这些区域的特征进行分类回归，其网 络框架如图 2 所示. 该类方法包含 R-CNN ${ }^{[20]}$, $\mathrm{SPPNet}^{[21]}$ Fast R-CNN ${ }^{[22]}$ Faster R-CNN, R-FCN ${ }^{[23]}$, Mask R-CNN ${ }^{[24]}$, Light-Head R-CNN ${ }^{[25]}$, Cascade R-CNN ${ }^{[26]}$ 和 TridentNet ${ }^{[27]}$ 等. 相比单阶段 检测网络，这类方法更注重目标检测的精度.

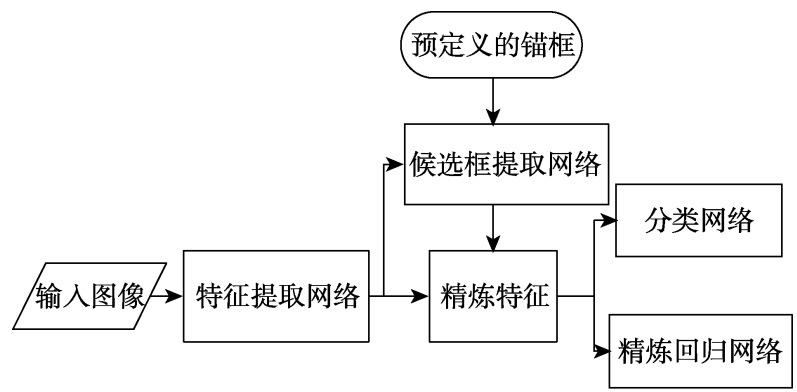

图 2 双阶段目标检测网络框架

以 Cascade R-CNN 为例，它通过为级联的 R-CNN 设置不同的交并比(intersection over union, IoU) 阈值, 逐步提高网络输出的精度. 该网络是通 用目标检测方法中检测性能最先进的方法之一. 但真实有雾场景下的目标可能与通用目标检测方 法所应用场景下的目标具有不同的分布, 将它直 接应用于真实有雾场景, 网络的性能会因此而降 低. 因此, 本文就目标分布对目标检测网络性能的 影响进行探讨, 即讨论知识引导如何促使通用目 
标检测网络更好地适应真实有雾场景.

\section{3 有雾场景下的目标检测方法}

目前，有雾场景下的目标检测方法主要可以 分为 2 类，一类是两阶段方法，即基于去雾-检测 的无关联目标检测方法. 该方法先使用图像增强 和恢复方法对有雾图像进行去雾, 然后对去雾图 像使用目标检测方法进行检测，其中第 1 阶段的去 雾过程可能给图像带来伪影、颜色失真和雾的残留 等问题，因此并非对所有使用了去雾方法的图像 进行检测都会提升检测精度. 另一类是端到端方 法，即将去雾网络和目标检测网络进行联合优化 训练, 同时进行去雾任务和检测任务. 目前该方法 仅有文献 [1]做过相关实验，其将去雾网络 AODNet 和检测网络 Faster R-CNN 进行端到端联 合优化，从而有效地提高合成的有雾场景下的目 标检测精度，但是合成的有雾图像与真实世界收 集的图像往往存在较大出人. 合成的图像上可能 存在视觉伪影以及与现实世界场景不符的有雾场 景. 本文认为模型联合优化对真实有雾场景下的 目标检测具有促进作用，并在下文探讨模型联合 优化如何提升真实有雾场景下目标检测的性能.

基于上述 3 个相关工作，本文从 2 个分支探讨 如何促进真实有雾场景下目标检测的研究. 一是 有雾场景目标检测数据集的构建与处理; 二是真 实有雾场景下目标检测的解决方案. 下文将对这 2 个分支进行展开讨论.

\section{2 有雾场景目标检测数据集的构建与处理}

收集大型的真实有雾场景下的目标检测数据 集是高耗时、高成本的工作，而当前已有较完备的 目标检测数据集，因此很自然地想到一种节约时间 及成本的方法：在目标检测数据集上合成有雾场 景. 考虑 $\mathrm{MS} \mathrm{COCO}$ 目标数量多、类别全且较为平 衡，场景丰富同时拥有干净、质量高的标注信息， 因此选择在 MS COCO 上造雾. 现有的图像去雾数 据集大多数是人工合成的有雾图像，因此已存在一 套完整的方法流程. 本文参考已有方法并设计了一 套系统化的合成有雾场景目标检测数据集的方法. 首先输人清晰图像，通过深度估计生成对应的深度 图; 接着, 为了减少合成的有雾图像存在与现实场 景不符的情况，采用导向滤波对深度图进行平滑; 最后通过大气散射模型, 将清晰图像和平滑后的深 度图进行合成, 得到最终的有雾场景目标检测数据 集 S-COCO. 本文方法流程如图 3 所示.

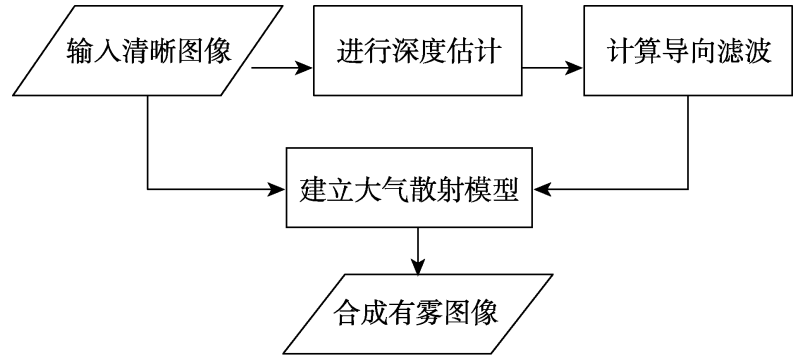

图 3 S-COCO 的系统化合成方法流程

\section{1 深度估计}

自然场景下的雾随景深的变化而分布不均, 为了获得更符合真实场景的有雾图, 需要使用有 效可靠的深度估计算法生成深度图. 本文采用自 监督单目深度估计挖掘框架 ${ }^{[28]}$ 对 MS COCO 中的 图像进行深度估计. 其使用竞争协作机制对单一 视角的深度估计、相机位姿估计、光流估计以及静 态场景和移动区域的分割这 4 个网络联合训练，充 分调动了各个网络之间的协作能力，进行精确的 深度估计；部分深度图效果如图 4 所示.

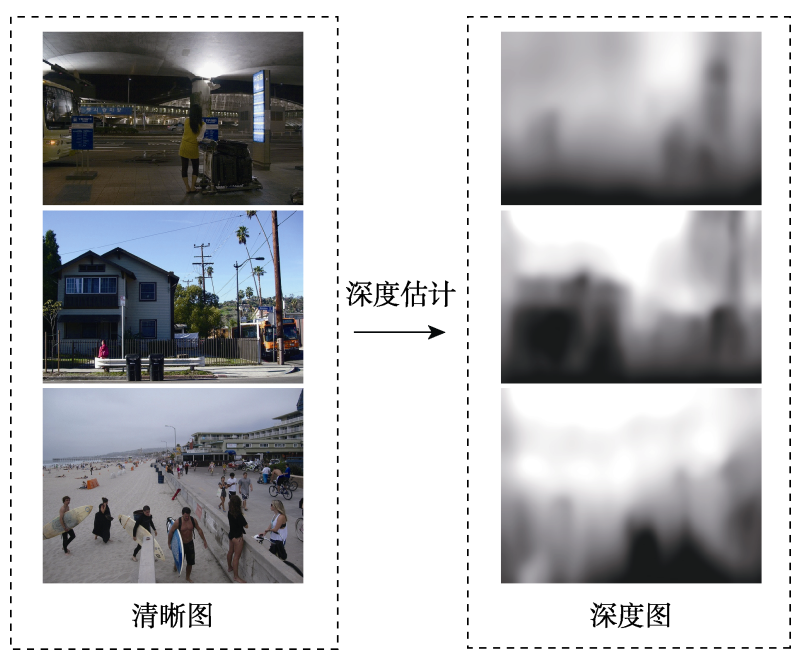

图 4 部分深度图效果示例

\section{2 导向滤波}

由于生成的深度图像可能受到块效应和噪声 等影响, 与实际深度图像之间存在一定的差异, 本 文采用导向滤波方法对深度图像进行平滑, 减少 深度图与清晰图像合成时产生背离现实场景的现 象，生成视觉上更加可信的有雾图像. 导向滤波的 公式定义为

$$
q_{i}=\frac{1}{|w|} \sum_{k: i \in w_{k}}\left(a_{k} I_{i}+b_{k}\right)
$$

其中, $i$ 为图像上 $i$ 位置的像素点; $q$ 为输出图像; $w_{k}$ 为一个以 $r$ 为半径的确定的窗口; $|w|$ 为窗口 $w_{k}$ 中像素点的个数; $I$ 为引导图像; $\left(a_{k}, b_{k}\right)$ 为唯 
一确定的常量系数，计算式分别为

$$
\begin{gathered}
a_{k}=\frac{\frac{1}{|w|} \sum_{i \in w_{k}} I_{i} p_{i}-\mu_{k} \bar{p}_{k}}{\sigma_{k}^{2}+\epsilon} \\
b_{k}=\bar{p}_{k}-a_{k} \mu_{k}
\end{gathered}
$$

其中, $\mu_{k}$ 和 $\sigma_{k}^{2}$ 分别为引导图像 $I$ 在窗口 $w_{k}$ 中的 平均值和方差; $\bar{p}_{k}=\frac{1}{|w|} \sum_{i \in w_{k}} p_{i}$ 表示输人图像 $p$ 在 窗口 $w_{k}$ 中的平均值; $\epsilon$ 为正则化参数, 避免 $a_{k}$ 过 大. 在平滑深度图时, 将半径 $r$ 和正则化参数 $\epsilon$ 分 别设置为 16 和 0.8 .

\section{3 大气散射模型}

大气散射模型一直是有雾图像生成的经典模 型. 根据大气散射模型将清晰图像和经过导向滤 波后的深度图进行合成, 得到最终的有雾场景目 标检测数据集 S-COCO. 大气散射模型定义为

$$
I(x)=\mathrm{e}^{-\beta d(x)} J(x)+A\left(1-\mathrm{e}^{-\beta d(x)}\right)
$$

其中, $I(x)$ 为观察到的有雾图像, 即需要合成的图 像; $J(x)$ 为清晰图像, 即 MS COCO 数据集中的图 像; $A$ 为地球大气亮度; $\beta$ 为大气层散射系数; $d(x)$ 为 MS COCO 数据集的深度图.

为了能够生成和真实有雾场景下相近的场景 分布，即生成不同浓度的雾场景，随机选择 $[0.7$, 1.0]之间的数均匀地分配给大气亮度 $A$ 的 3 个通 道，同时令 $\beta \in[0.6,1.8]$. 最终合成的 S-COCO 包 含 7721 幅带雾图像、清晰图像以及 5 类目标检测 标签信息, 生成的有雾图像如图 5 所示. 从图 5 中 可以看出, 当雾比较浓时, 合成的有雾图像的块效 应较为明显; 当雾比较薄时, 合成图像更接近真实 有雾场景下拍摄的图像.

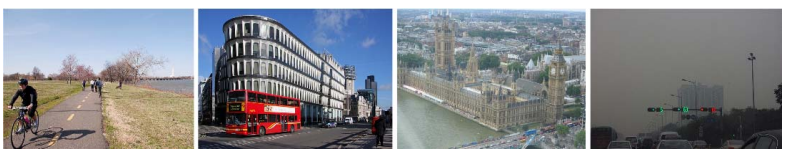

a. S-COCO 清晰图像示例

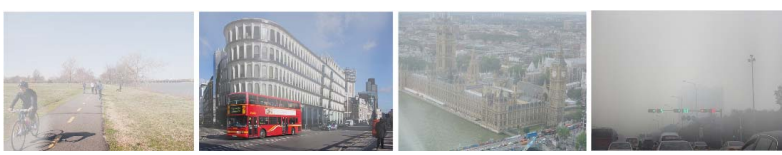

b. S-COCO 有雾图像示例

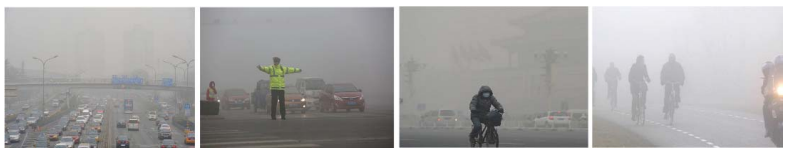

c. RTTS 图像示例

图 5 S-COCO 和 RTTS 图像示例

\section{4 数据集处理}

真实有雾场景下的目标检测数据集存在采集 难、标注难的特点, 因此在标注时很容易引入脏标 签. 对比现存的 RTTS 和 UG-test 数据集, 可以观 察到收集到的数据集存在漏标、错标的情况，同时 不同数据集的标注标准存在偏差. 例如, RTTS 中 卡车被错误地标注为汽车, 而 UG-test 数据集中汽 车标注均正确. 因此, 将这 2 个数据集分别作为训 练集和测试集时，模型的性能会因为这些噪声的 存在而大大降低. 为了提高模型的检测能力, 针对 RTTS 数据集进行数据清洗, 将 2 个数据集的标注 规则进行统一，同时模型还需要相应的后处理.

通过合成有雾场景下目标检测数据集的构建 和真实有雾场景下目标检测数据集的处理, 可以 有效地提升真实有雾场景下的目标检测器性能, 下文实验将验证该方法的有效性.

\section{3 方法概述}

通过对真实有雾场景下的目标检测问题的分 析, 本文针对知识引导的作用提出了基于知识引 导的目标检测方法 KODNet; 针对模型联合优化的 作用提出了图像去雾和目标检测的联合学习框架 DONet. KODNet 旨在解决有雾场景下目标检测数 据集中目标分布偏移问题，通过统计先验设计检 测网络中的针框面比, 从而指导有雾场景下的目 标检测. DONet 旨在解决基于去雾-检测的无关联 目标检测方法中所存在的问题，即直接使用图像 增强与恢复方法时会使图像产生伪影、细节缺失和 颜色失真等问题，从而影响下游检测任务的精度. DONet 通过将去雾网络和检测网络联合优化，使 网络学习图像去雾中恢复的结构细节特征和颜色 特征，从而提高检测网络的精度.

\section{1 基于知识引导的目标检测}

由于真实有雾场景数据集的缺少, 为训练出 鲁棒且效果好的模型，通常需要使用其他合成的 有雾数据集, 从而减少无雾场景带来的偏移. 然而 这种操作会造成源域(即训练集)和目标域(即测试 集)之间目标分布偏移的问题, 如图 6 所示. 直接 使用通用目标检测器对有雾场景的目标进行训练, 必然会因不能选取准确的候选区域而影响目标特 征的学习, 最终导致检测器性能的下降. 基于这个 问题，提出了基于知识引导的目标检测框架，通过 统计先验设计检测网络中的针框面比, 从而指导 网络学习该场景下目标的特征. 


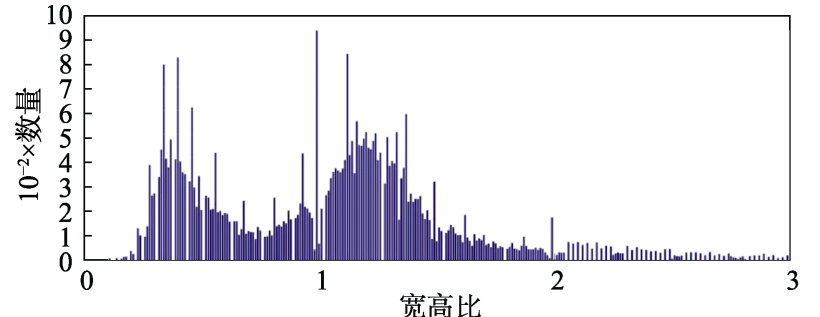

a. $\mathrm{S}-\mathrm{COCO}$

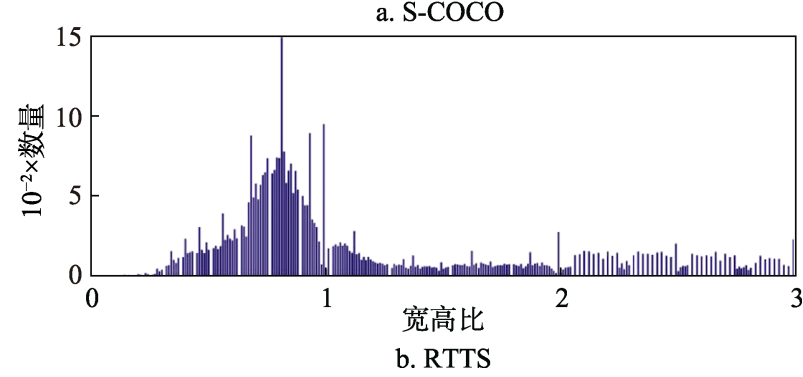

图 6 S-COCO 和 RTTS 目标宽高比分布

\section{1 .1 框架流程}

以通用目标检测网络为 Cascade R-CNN 的 KODNet 为例详细描述网络流程, 网络结构如图 7 所示. 输人图像首先进人主干网络进行目标特征 提取并将提取到的特征图送人 RPN. 在 RPN 中, 通过预设的针框面比、尺度以及阈值生成大量的候 选区域. 接着将这些候选区域送人级联的 R-CNN
结构, 通过级联的感兴趣区域(region of interest, RoI)对齐层提取候选区域的特征, 并将 RoI 特征采 样成统一大小的特征图后进行分类和回归.

\section{1 .2 针框设计}

针对通用目标检测网络中的针框，提出了使 用先验统计对其进行设计, 从而使网络学习更多 的目标特征. 假设需要预测的特征图大小为 $m \times n$, 将每个像素点作为生成的针框中心, 并为每个中 心预设需要生成的针框形状, 称为针框面比. 在针 框面比的设计中，不使用手工挑选的方法，而是使 用先验统计方法 $-k$-means 聚类获取针框面比大 量分布的区域，使其尽可能覆盖所有目标，以此提 升检测器的检测性能. 具体做法是计算所有目标 的面比并将其作为样本. 其中, 设置 $k=4$ 进行标准 的 $k$-means 操作. 聚类时, 首先从样本中随机选择 $k$ 个框的面比作为质心，然后对每个样本计算其 与质心的欧几里得距离, 将距离质心最近的样本 归类为一个簇. 最终聚类得到的簇的质心即为该 场景下的目标形状信息. 以簇的质心作为检测器 采样框的面比, 可以使 RPN 生成大量覆盖在目标 区域的针框，有效地利用正样本的知识，学习更为 准确的目标特征.

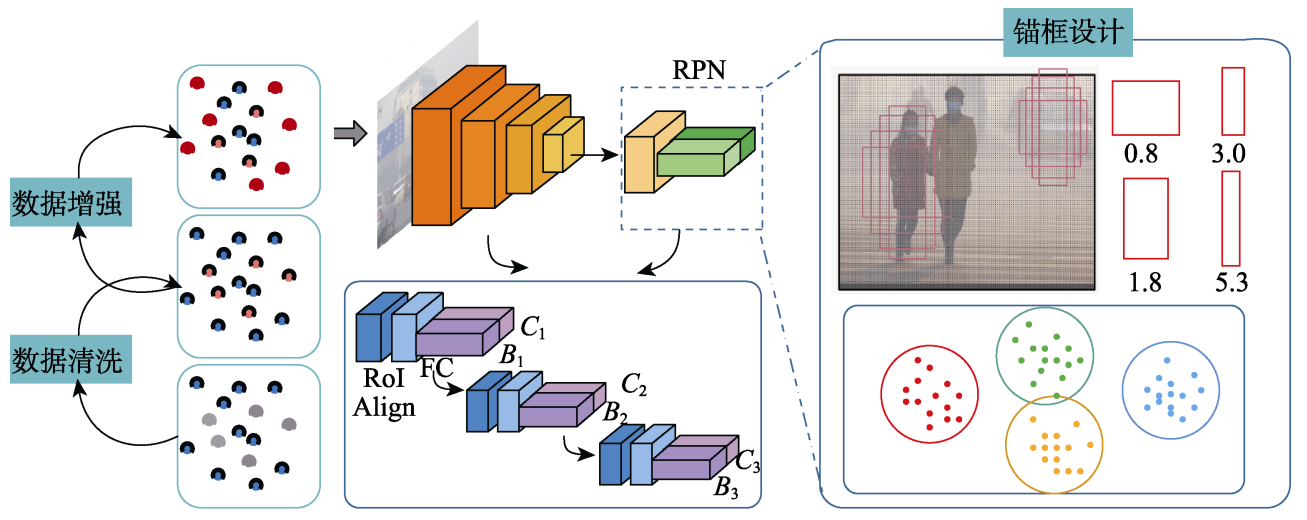

图 7 KODNet 网络结构

\section{1 .3 数据增强}

在真实有雾场景下，空气中的雾、尘埃和烟等 物质造成拍摄的图像模糊、光照异常和颜色失真等 问题. 与真实有雾场景相比，合成的有雾场景目标 检测数据集仍然与之存在差异，如存在伪影、光照 条件与雾天不符以及目标外观存在差异等. 为了 丰富图像的场景，本文采用数据增强的方法增加 样本的多样性, 以提高网络的泛化能力. 其中针对 有雾场景进行了一系列光度失真的数据增强操作, 使检测器更好地适应有雾场景。

\section{2 基于模型联合优化的目标检测}

基于去雾-检测的无关联目标检测方法，即先 使用图像去雾方法对图像进行去雾, 再对去雾后 的图像进行检测。这种方法会使图像在去雾过程 中产生伪影、细节缺失和颜色失真等问题，从而影 响下游检测任务的精度. 基于这些问题提出 DONet，通过将去雾网络和检测网络联合优化，图 像恢复的结果会在下游检测信息的引导下进行重 构，同时检测网络可以学习图像去雾中恢复的结构 细节特征和颜色特征，从而提高检测网络的精度. 


\subsection{1 框架流程}

模型联合优化框架是一个端到端的多任务学 习框架, 其中包括图像去雾任务、分类任务以及回 归任务, 如图 8 所示. 模型联合优化框架包括去雾 网络模块、检测网络模块以及联调学习的损失函 数. 其中去雾网络模块为当前主流的基于深度学 习的去雾网络(图 8 中去雾网络模块表示其中一种 去雾网络结构), 检测网络模块表示当前主流的目 标检测网络.

模型联合优化框架有 3 个输人，分别是有雾图 像、相应的清晰图像以及目标检测标签. 有雾图像 和相应的清晰图像将应用于去雾网络模块, 进行 图像去雾过程的学习以及去雾损失的计算, 并在
去雾模块末端得到 1 幅去雾图像. 接着将去雾后的 图像以及图像的标签送人检测网络模块, 进行目 标检测器的训练. 检测网络模块末端将输出目标 的分类置信度以及位置的偏移量

\subsection{2 网络实例}

以去雾网络为 PFFNet 和检测网络为 Faster R-CNN 的 DONet 为例, 对网络进行具体描述. PFFNet 是一个端到端的图像去雾网络, 它将渐进 特征融合的 U-Net ${ }^{[29]}$ 作为深度网络, 从有雾图像直 接转换到无雾的图像而不需要任何特殊的中间图 像. Faster R-CNN 是一个端到端的目标检测网络, 通过提出的 RPN 优化建议区域的生成方式, 实现 了端到端的高精度目标检测。

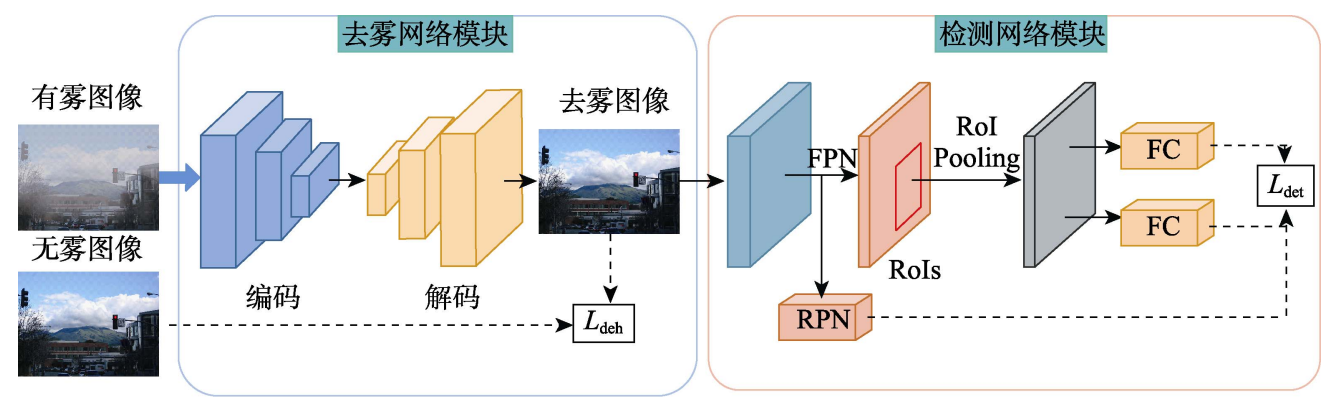

图 8 DONet 网络结构

PFFNet 使用均方误差函数(mean square error, MSE)作为其网络损失，定义为

$$
L_{\mathrm{MSE}}(\hat{X}, Y)\|\phi(\hat{X})-\phi(Y)\|_{2}
$$

其中， $\hat{X}$ 表示去雾后图像; $Y$ 表示清晰图像; $\phi(\cdot)_{i}$ 表示 VGG19 网络的第 $i$ 个卷积阶段生成的特征图; $\|\cdot\|_{2}$ 表示 2 范数.

Faster R-CNN 的任务分别是目标的分类和位 置的回归，因此该网络的损失由 2 部分损失组成, 定义为

$$
L_{\mathrm{det}}=\lambda_{a} L_{\mathrm{cls}}+L_{\mathrm{reg}}
$$

其中, $L_{\mathrm{cls}}$ 和 $L_{\mathrm{reg}}$ 分别为分类损失和回归损失, 分 类损失一般使用交叉熵损失函数，回归损失一般 使用 smooth $L_{1}$ 损失函数; $\lambda_{a}$ 为平衡因子.

对于 PFFNet 和 Faster R-CNN 联合优化的网 络, 输人分别为有雾图像、清晰图像和检测标签, 同时不对图像做任何增强操作。在 PFFNet 中, 有 雾图像经过特征编码和特征解码后得到去雾图像. 同时根据式(5)计算去雾网络损失函数 $L_{\mathrm{deh}}$. 接着 将去雾后图像送人 Faster R-CNN 进行目标检测任 务的学习, 最后网络输出目标所属的类别以及相 应的位置. 检测网络损失函数如式(6)所示.

最后 DONet 的整体损失定义为去雾网络损失
函数与检测网络损失函数加权和, 即

$$
L=\lambda L_{\mathrm{deh}}+L_{\mathrm{det}}
$$

其中, $L$ 为 $\mathrm{DONet}$ 的整体损失函数; $L_{\mathrm{deh}}$ 为去雾 网络模块损失; $L_{\mathrm{det}}$ 为检测网络模块损失; $\lambda$ 为平 衡因子.

在后续实验中，将以不同去雾网络和目标检 测网络两两组合的方式验证 DONet 在真实有雾场 景下进行目标检测的有效性.

\section{4 实验结果与分析}

\section{1 数据集和实验设置}

本节设计了 3 类实验. (1) 真实有雾场景下的 目标检测评估. 为了验证知识引导和联合优化对 真实有雾场景下目标检测性能的促进作用，分别 评估了 3 种真实有雾场景下的目标检测方法：基于 知识引导的目标检测、基于去雾-检测的无关联目 标检测和基于模型联合优化的目标检测。(2) 合成 有雾场景下的目标检测评估. 为了验证 DONet 在 合成有雾场景目标检测数据集的有效性，在 FogyCityscapes 数据集上对其评估. (3) 数据集处理对 真实有雾场景下目标检测的影响。 


\section{1 .1 数据集}

实验所用数据集包括 S-COCO, RTTS, UG-test, Cityscapes 以及 Foggy-Cityscapes ${ }^{[30]}$. 其中, Cityscapes 数据集包含来自 50 个不同城市的街景视频 序列, 实例标签共有 9 类. Foggy-Cityscapes 数据集
是一个合成的有雾数据集, 其合成图像是由 Cityscapes 提供的图像和深度图进行渲染所得. Foggy-Cityscapes 的标签以及数据集的划分和 Cityscapes 相同. 实验所用数据集包含的类别、目 标数、图像数量以及图像性质如表 1 所示.

表 1 实验所用数据集统计结果

\begin{tabular}{|c|c|c|c|c|c|c|c|c|c|c|}
\hline \multirow{2}{*}{ 数据集 } & \multicolumn{8}{|c|}{ 类别 } & \multirow{2}{*}{ 图像数 } & \multirow{2}{*}{ 图像性质 } \\
\hline & 汽车 & 自行车 & 摩托车 & 人 & 公共汽车 & 骑手 & 火车 & 卡车 & & \\
\hline $\mathrm{S}-\mathrm{COCO}$ & 13009 & 4927 & 6420 & 30303 & 5241 & & & & 7721 & 合成 \\
\hline $\operatorname{RTTS}^{[3]}$ & 25317 & 698 & 1232 & 11366 & 2590 & & & & 4322 & 真实 \\
\hline UG-test $^{[5]}$ & 18074 & 225 & 3804 & 1562 & 536 & & & & 2987 & 真实 \\
\hline Foggy-Cityscapes $^{[30]}$ & 32728 & 5442 & 892 & 21864 & 477 & 2306 & 191 & 575 & 3450 & 合成 \\
\hline
\end{tabular}

\subsection{2 实验设置}

本文方法实验环境详细信息如下：软件环境 为 64 位 Ubuntu16.04, CUDA 版本为 9.0.176, CUDNN 版本为 7.6.0.64; 硬件设备为 TITAN Xp, GPU 显存为 $12 \mathrm{~GB}$, 实验皆在基于 Pytorch1.0.1 深 度学习平台的 MMDetection ${ }^{[31]}$ 目标检测工具箱上 进行. 测试和训练时均对输人图像随机调整短边 尺度到 800 , 另外一条边则根据宽高比调整. 所有 实验的评估指标均为均值平均精度(mean average precision, $\mathrm{mAP})$ ，其 $\mathrm{IoU}$ 阈值设为 0.5 .

\section{2 真实有雾场景下目标检测的评估}

本节实验以 S-COCO 数据集为训练集, RTTS 数据集为测试集，旨在评估本文方法在真实有雾 场景下进行目标检测的精度. 训练时初始学习率 为 0.00125 , 动量衰减参数分别设置为 0.9 和 0.0001 . 训练总轮数为 15 轮, 学习率在第 8 轮和第 11 轮之 后衰减为原来的 $10 \%$.

\subsection{1 基于知识引导的目标检测的评估}

以通用目标检测方法 Cascade R-CNN 为例设 计 3 个实验. 第 1 个实验使用 Cascade R-CNN 在合 成的数据集 S-COCO 进行微调训练, 其中针框面 比为 $0.5,1.0$ 和 2.0. 第 2 个实验使用本文的 KODNet 在 S-COCO 数据集上进行微调训练, 根据统计先 验知识, 将针框面比设置为 $0.8,1.8,3.0$ 和 5.3. 第 3 个实验在第 2 个实验的基础上对训练数据进行光 度失真等一系列的数据增强操作. 其中, 3 个实验所 用的主干网络均为 ResNeXt-101(64×4d $)^{[32]}$ 和 $\mathrm{FPN}^{[33]}$.

表 2 展示了 KODNet 在 RTTS 上的评估结果. 第 1 行为 Cascade R-CNN 在 S-COCO 上进行微调 训练后的检测结果. 第 2 行为使用知识引导的 Cascade R-CNN 的实验结果，可以看到本文提出的
KODNet 相对于通用目标检测器精度提升了 $2.8 \%$. 第 3 行为使用数据增强的 KODNet 的检测结果, 可 以看出使用数据增强后, 该网络精度可以达到 $70.5 \%$ ，与 KODNet 相比精度提升了 $3.6 \%$. 因此, 使用先验统计知识提前获取框的知识, 并使用光 度失真等数据增强操作对数据集处理, 有助于检 测器学习该场景下目标的形状、个体外观和颜色等 信息.

表 2 KODNet 在 RTTS 上的评估结果 \%

\begin{tabular}{lcccccc}
\hline \multirow{2}{*}{ 方法 } & \multicolumn{6}{c}{ 类别 } \\
\cline { 2 - 6 } & 公共汽车 & 自行车 & 汽车 & 摩托车 & 人 & \\
\hline Cascade R-CNN & mAP \\
KODNet & 79.1 & 46.0 & 75.2 & 58.6 & 60.9 & 64.1 \\
KODNet+DA & 80.0 & 48.9 & 74.5 & 64.3 & 66.6 & 66.9 \\
\hline
\end{tabular}

\subsection{2 基于去雾-检测的无关联目标检测的评估}

首先使用现有的检测性能较好的目标检测模 型直接对有雾图像进行评估, 接着使用基于去雾检测的无关联目标检测方法，对图像先去雾再使 用通用目标检测方法对去雾后的图像进行评估. 其中目标检测方法包括单阶段目标检测方法 RetinaNet、双阶段目标检测方法 Faster R-CNN 和 Cascade R-CNN, 图像去雾方法包括 AODNet, PFFNet 和 HI-DehazeNet ${ }^{[34]}$.

测试 RTTS 数据集中所有目标, 包括被标注为 “困难”的样本. 评估所用的检测模型是各个检测 器在 MMDetection 平台上针对 MS COCO 训练好 的模型, 并且不对模型进行任何微调. 其中, RetinaNet 和 Faster R-CNN 采用 ResNet-101 ${ }^{[35]}$ 以及 FPN 作为其主干网络, Cascade R-CNN 采用 ResNet-50 以及 FPN 作为其主干网络. 
表 3 展示了上述 2 种实验设置的评估结果. 可 以看出, 直接对原始图像进行测试时, RetinaNet 网 络的检测结果最好. 由于 Cascade R-CNN 使用的 主干网络为 ResNet-50, 所以检测结果比 RetinaNet 网络低 3.4\%. 而对图像先进行去雾操作, 再使用 检测器进行评估的精度几乎都下降了. 其中, 在 RetinaNet 检测器上对使用了去雾方法的图像进行
测试, 结果均大幅度下降. 而使用其余 2 个检测器 对去雾图像进行测试的提升效果为 $-2.3 \% \sim 0.5 \%$. 可见，尽管图像去雾效果在人眼观察上是明显的， 但检测器并不认可图像去雾的效果. 这是因为去 雾后的图像存在结构细节缺失以及颜色失真等问 题, 同时可能引人了不利于检测的噪声, 致使检测 器的性能下降.

表 3 基于去雾-检测的无关联目标检测方法在 RTTS 的评估结果

$\%$

\begin{tabular}{|c|c|c|c|c|c|c|c|}
\hline \multirow{2}{*}{\multicolumn{2}{|c|}{$\frac{\text { 无关联目标检测方法 }}{\text { 检测-去雾 }}$}} & \multicolumn{5}{|c|}{ 类别 } & \multirow{2}{*}{ mAP } \\
\hline & & 公共汽车 & 自行车 & 汽车 & 摩托车 & 人 & \\
\hline RetinaNet $^{[17]}$ & & 82.6 & 60.0 & 73.9 & 60.1 & 46.2 & 64.5 \\
\hline \multirow{3}{*}{ RetinaNet $^{[17]}$} & $\mathrm{AODNet}^{[1]}$ & 71.7 & 50.5 & 53.5 & 33.9 & 25.7 & 47.1 \\
\hline & $\mathrm{PFFNet}^{[9]}$ & 71.8 & 49.7 & 53.7 & 42.3 & 25.8 & 48.6 \\
\hline & HI-DehazeNet ${ }^{[34]}$ & 71.8 & 49.9 & 53.8 & 41.3 & 26.0 & 48.5 \\
\hline \multicolumn{2}{|l|}{ Faster R-CNN ${ }^{[2]}$} & 83.6 & 56.3 & 73.0 & 59.6 & 43.3 & 63.1 \\
\hline \multirow{3}{*}{ Faster R-CNN ${ }^{[2]}$} & $\mathrm{AODNet}^{[1]}$ & 82.4 & 56.0 & 70.8 & 58.0 & 41.5 & 61.7 \\
\hline & $\mathrm{PFFNet}^{[9]}$ & 82.4 & 56.6 & 72.6 & 60.2 & 42.8 & 62.9 \\
\hline & HI-DehazeNet ${ }^{[34]}$ & 83.1 & 56.7 & 74.0 & 58.8 & 42.7 & 63.1 \\
\hline \multicolumn{2}{|l|}{ Cascade R-CNN ${ }^{[26]}$} & 79.7 & 56.8 & 74.1 & 57.1 & 37.8 & 61.1 \\
\hline \multirow{3}{*}{ Cascade R-CNN ${ }^{[26]}$} & $\mathrm{AODNet}^{[1]}$ & 72.9 & 55.5 & 71.9 & 51.8 & 35.9 & 58.8 \\
\hline & $\mathrm{PFFNet}^{[9]}$ & 79.3 & 58.1 & 73.1 & 59.4 & 37.4 & 61.5 \\
\hline & HI-DehazeNet ${ }^{[34]}$ & 79.6 & 58.3 & 74.9 & 56.5 & 38.5 & 61.6 \\
\hline
\end{tabular}

注. 粗体表示使用相同检测器进行评估得到的最好的检测精度. 检测-去雾表示先使用去雾方法，再使用检测方法.

图 9 展示了基于去雾-检测的无关联目标检测 方法在 RTTS 上进行评估的可视化结果. 其中, 第 1 列所示图像是使用 Cascade R-CNN 在 MS COCO 数据集上训练好的模型进行测试得到的可视化结 果，可以看出，该模型的检测效果最好. 第 2 列、 第 3 列和第 4 列所示图像分别是使用 AODNet, PFFNet 和 HI-DehazeNet 对图像去雾后，再使用该 模型对去雾后图像进行检测的可视化结果. 从图 9 中可以看到，对去雾后图像进行检测的结果存在 更多错检和漏检的目标，这说明使用去雾方法对 图像进行去雾处理无法有效提升网络的检测性能.

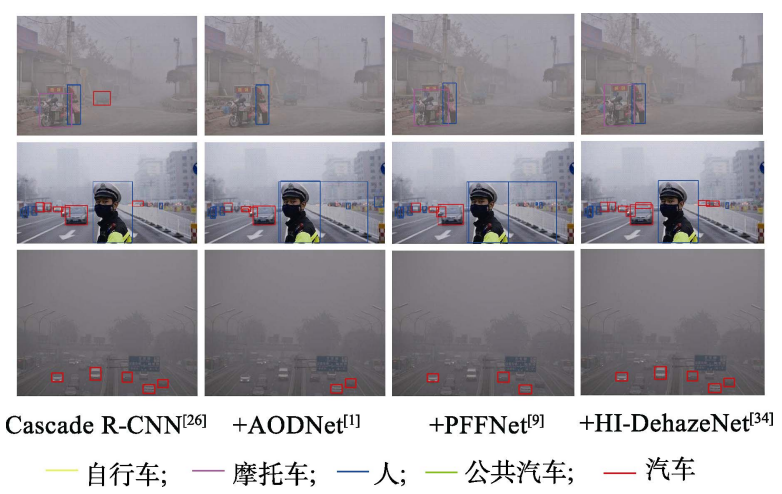

图 9 基于去雾-检测的无关联目标检测方法检测结果

\subsection{3 基于模型联合优化的目标检测的评估}

本节实验将不同的去雾方法和不同的检测方 法进行两两组合联合优化学习，从而探讨联合优化 对真实有雾场景下目标检测的有效性. 其中, 图像 去雾方法包括 AODNet, PFFNet 和 HI-DehazeNet, 目标检测方法包括 RetinaNet, Faster R-CNN 和 Cascade R-CNN. 其中 RetinaNet 和 Faster R-CNN 采用 ResNet-101 以及 FPN 作为其主干网络, Cascade R-CNN 采用 ResNet-50 以及 FPN 作为其主干 网络, 按照经验设置检测网络损失中的平衡因子 $\lambda_{a}=1$. 本文设置了 2 种实验方法: 使用目标检测 方法对 S-COCO 进行微调训练以及使用上述两两 组合的 DONet 网络在 S-COCO 上训练. 训练时, AODNet, PFFNet 和 HI-DehazeNet 网络损失函数前 的平衡因子 $\lambda$ 根据经验设置, 在本实验中分别设 置为 $0.1,10.0$ 和 10.0 .

表 4 展示了 3 个去雾网络和 3 个检测网络两两 组合后的 DONet 框架的检测结果. 通过表 4 可以 看出, 使用原始检测器对有雾图像直接训练得到 的模型中, RetinaNet的检测精度最高，达到 $63.0 \%$. 当使用 DONet 对有雾图像进行联合学习优化时, 除去雾网络为 AODNet 的 DONet 框架外, 其余框 
架的测试精度均有 $0.9 \%$ $4.7 \%$ 的提升. 其中, 使用 PFFNet 和 Faster R-CNN 的 DONet 框架训练出来的 模型检测性能最好, mAP 达到 $66.6 \%$.

尽管并非所有的 DONet 的检测性能都比通用 目标检测方法的检测性能更好，但是从实验的整 体来看, 去雾网络和检测网络的联合优化训练的 确可以有效地提升真实有雾场景下的目标检测性
能. 在 DONet 中, AODNet 和 RetinaNet 组合以及 AODNet 和 Cascade R-CNN 组合进行联合训练得 到的检测器性能都降低了. 一方面, AODNet 网络 的去雾能力相比于其余 2 个网络有所欠缺; 另一方 面, AODNet 去雾后存在更多的细节丢失和颜色失 真, 影响下游检测器的训练, 因此该去雾网络组成 的 DONet 的检测精度有所下降.

表 4 DONet 在 RTTS 的评估结果

\begin{tabular}{|c|c|c|c|c|c|c|c|}
\hline \multirow{2}{*}{\multicolumn{2}{|c|}{$\begin{array}{c}\text { DONet 方法 } \\
\text { 检测+去雾 } \\
\end{array}$}} & \multicolumn{5}{|c|}{ 类别 } & \multirow{2}{*}{$\mathrm{mAP}$} \\
\hline & & 公共汽车 & 自行车 & 汽车 & 摩托车 & 人 & \\
\hline RetinaNet $^{[17]}$ & & 78.4 & 44.2 & 70.8 & 58.3 & 63.2 & 63.0 \\
\hline \multirow{3}{*}{ RetinaNet $^{[17]}$} & AODNet $^{[1]}$ & 77.7 & 43.0 & 70.0 & 53.9 & 57.5 & 60.4 \\
\hline & $\mathrm{PFFNet}^{[9]}$ & 80.9 & 46.7 & 71.4 & 63.4 & 66.2 & 65.7 \\
\hline & HI-DehazeNet ${ }^{[34]}$ & 79.1 & 46.3 & 71.3 & 58.3 & 64.7 & 63.9 \\
\hline \multicolumn{2}{|l|}{ Faster R-CNN ${ }^{[2]}$} & 77.7 & 47.0 & 71.2 & 57.1 & 56.5 & 61.9 \\
\hline \multirow{3}{*}{ Faster R-CNN ${ }^{[2]}$} & $\mathrm{AODNet}^{[1]}$ & 78.9 & 48.1 & 71.0 & 56.2 & 59.2 & 62.7 \\
\hline & $\mathrm{PFFNet}^{[9]}$ & 80.5 & 51.6 & 74.0 & 62.7 & 64.3 & 66.6 \\
\hline & HI-DehazeNet ${ }^{[34]}$ & 80.0 & 47.9 & 73.1 & 60.9 & 62.8 & 64.9 \\
\hline \multicolumn{2}{|l|}{ Cascade R-CNN ${ }^{[26]}$} & 77.3 & 42.5 & 68.5 & 58.0 & 64.8 & 62.2 \\
\hline \multirow{3}{*}{ Cascade R-CNN ${ }^{[26]}$} & $\mathrm{AODNet}^{[1]}$ & 77.0 & 43.7 & 70.6 & 56.5 & 59.3 & 61.4 \\
\hline & $\mathrm{PFFNet}^{[9]}$ & 78.6 & 47.0 & 72.7 & 62.5 & 65.5 & 65.3 \\
\hline & HI-DehazeNet ${ }^{[34]}$ & 78.5 & 46.1 & 72.1 & 58.5 & 66.6 & 64.4 \\
\hline
\end{tabular}

注. 粗体表示使用相同检测器进行评估得到的最好的检测精度.

图 10 展示了 DONet 框架在 RTTS 数据集上进 行目标检测的部分可视化结果. 第 1 列所示图像是 使用 Cascade R-CNN 在 S-COCO 上进行微调训练 后的可视化结果. 第 2 4 列是分别使用 AODNet, PFFNet, HI-DehazeNet 和 Cascade R-CNN 联合训练 得到的模型结果. 从图 10 中可以明显看出, PFFNet 和 Cascade R-CNN 联合训练得到的模型的 检测结果最好. 通过去雾操作，本文方法可以通过 更多的细节检测出隐藏在雾后面的目标，从而提 高最终检测器的性能.
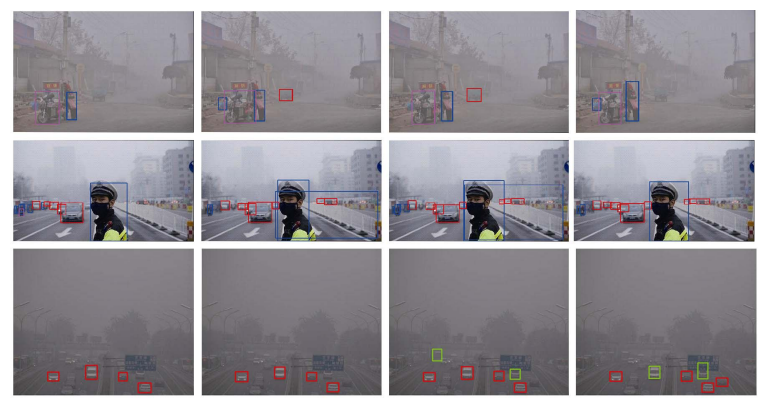

Cascade R-CNN ${ }^{[26]}+$ AODNet $^{[1]}$

+ PFFNet $^{[9]} \quad+$ HI-DehazeNet ${ }^{[34]}$ 自行车; 一摩托车; 一人; 一公共汽车; 一汽车

图 10 DONet 在 RTTS 的检测结果

\section{3 合成有雾场景下目标检测的评估}

真实世界采集的有雾图具有多种降质因素， 而合成的有雾图像是根据物理模型生成, 雾更加 均匀且单一. 本节实验将在 Foggy-Cityscapes 数据 集上进行，其中 Foggy-Cityscapes 是合成的有雾图 像. Foggy-Cityscapes 存在 3 种不同浓度的雾, 在大 气散射模型中对应的 $\beta$ 值分别为 $0.005,0.010$ 和 0.020. 随机挑选一种浓度的雾与原图合成有雾图, 以达到在现实场景下收集数据集的效果. 实验时 将 Foggy-Cityscapes 数据集中的训练集用于模型训 练, 将验证集作为测试集用于模型性能的评估. 本 节实验中, 以 AODNet, PFFNet 和 HI-DehazeNet 为 去雾网络, Faster R-CNN 为检测网络, 分别两两组合 进行联合优化训练. 训练时初始学习率为 0.00125 , 动量衰减参数分别设置为 0.9000 和 0.0001 . 训练 总轮数为 15 轮, 学习率在第 8 轮和第 11 轮之后衰 减为原来的 0.1 .

表 5 展示了 DONet 使用 Faster R-CNN ${ }^{[2]}$ 检测方 法在 Foggy-Cityscapes 验证集上的评估结果. 可以 看出，使用 HI-DehazeNet 和 Faster R-CNN 进行联合 学习得到的检测器性能比直接使用目标检测方法进 
表 5 DONet 在 Foggy-Cityscapes 的评估结果

\begin{tabular}{|c|c|c|c|c|c|c|c|c|c|c|}
\hline \multirow{2}{*}{\multicolumn{2}{|c|}{$\begin{array}{c}\text { DONet 方法 } \\
\text { 检测+去雾 }\end{array}$}} & \multicolumn{8}{|c|}{ 类别 } & \multirow{2}{*}{$\mathrm{mAP}$} \\
\hline & & 公共汽车 & 自行车 & 汽车 & 摩托车 & 人 & 骑手 & 火车 & 卡车 & \\
\hline \multicolumn{2}{|l|}{ Faster R-CNN ${ }^{[2]}$} & 51.4 & 51.7 & 70.3 & 41.9 & 50.9 & 55.4 & 27.1 & 35.0 & 48.0 \\
\hline \multirow{3}{*}{ Faster R-CNN ${ }^{[2]}$} & $\mathrm{AODNet}^{[1]}$ & 52.4 & 49.4 & 69.5 & 36.8 & 48.6 & 53.7 & 27.4 & 34.4 & 46.5 \\
\hline & $\mathrm{PFFNet}^{[9]}$ & 51.3 & 50.5 & 69.2 & 42.9 & 48.3 & 54.7 & 27.3 & 29.2 & 46.7 \\
\hline & HI-DehazeNet ${ }^{[34]}$ & 51.6 & 51.4 & 70.0 & 42.2 & 50.4 & 56.5 & 31.9 & 38.1 & 49.0 \\
\hline
\end{tabular}

注. 粗体表示使用相同检测器进行评估得到的最好的检测精度.

行训练得到的检测器性能提升了 $1 \%$. 使用 AODNet 和 PFFNet 的 DONet 在该合成数据集上的检测精度 有所下降, 这是因为与 HI-DehazeNet 相比, AODNet 和 PFFNet 在该数据集上的去雾效果较差, 存在更 多的细节丢失，致使下游的检测性能下降.

\section{4 数据集处理对目标检测的影响}

本节实验旨在验证数据集处理对提升真实有 雾场景下目标检测性能的有效性，为今后该方向 的研究提供一个解决思路. 选取 KODNet 作为实 验方法在 RTTS 上训练, 并在 UG-test 上进行评估.

\subsection{1 数据集处理}

通过观察 RTTS 和 UG-test 数据集目标的标注 情况，发现这 2 个数据集的标注规则不同. 为此, 针对 RTTS 数据集进行数据清洗, 将 2 个数据集的 标注规则进行统一. RTTS 数据集的清洗规则为: （1）对未标注的摩托车重新标注; (2) 将骑车的人 的标签修改标注为骑手; (3) 将类别为卡车但标注 为公共汽车的标签修改为卡车. 数据清洗前后图 像标签变化如图 11 所示. 在数据清洗之后, RTTS 标签成为 7 类, 分别是人、公共汽车、汽车、摩托 车、自行车、卡车和骑手. 在训练时使用这 7 类标 签，而测试时仍然使用原始的 5 类标签.

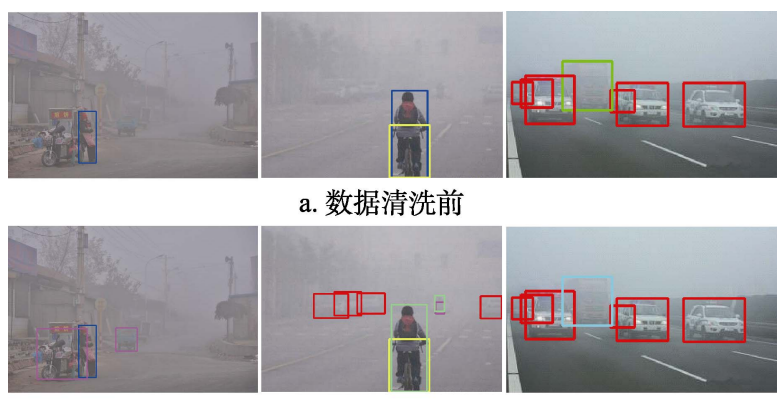

b. 数据清洗后

一自行车; 一 摩托车; 一人; 一 公共汽车; 一汽车;一骑手; 一卡车

图 11 数据清洗前后图像对比
此外, UG-test 数据集中类别为自行车的框高 与类别为人的框高相同，因此在测试时进行后处 理: 将自行车类别的框压缩为原本框高度的一半.

4.4.2 实验设置

实验以主干网络为 ResNet-101(64×4d) 和 FPN 的 Cascade R-CNN 为例设计了 5 个实验. (1) 使用 Cascade R-CNN 在 MS COCO 上的实验配置对 RTTS 进行训练, 并将在 UG-test 上测试的结果作 为基准. (2) 使用本文提出的针框设计方法对针框 面比进行设置, 针框面比设置为 $0.8,1.7,2.6$ 和 3.7. (3) 在 RTTS 数据集进行数据清洗并使用实验(2)的 设置进行训练. (4) 在上一实验设置的基础上对图 像进行光度失真等一系列数据增强操作. (5) 在实 验(4)训练的模型进行测试时, 使用后处理操作. 由于 UG-test 只公开 100 幅图像, 因此测试时只在 这 100 幅图像上进行评估.

表 6 展示了 KODNet 使用上述实验设置进行 训练得到的模型的测试结果. 由表 6 可见，基准方 法的 $\mathrm{mAP}$ 仅为 $28.63 \%$, 使用统计先验方法对针框 设计后, 网络的 $\mathrm{mAP}$ 提升 $2.23 \%$. 由此可以看出, 本文提出的 KODNet 可以缓解因域不同而造成的 目标分布偏移问题. 对 RTTS 进行数据清洗后, KODNet 训练的模型的精度达到 $42.17 \%$ ，与只使 用针框设计相比, $\mathrm{mAP}$ 提升 $11.31 \%$. 从表 6 可以看 出, 最终使用数据清洗、数据增强和后处理操作的 KODNet 精度达到了 $54.79 \%$, 性能得到了明显改 善, 可见具有无噪声、高质量的标注信息对提升检 测器性能的重要性.

表 7 展示了本文方法和 IEEE CVPR2019 $\mathrm{UG}^{2}+$ 挑战赛排行榜 ${ }^{\circledR}$ 上的选手所设计方法的对比结果. 评估结果在 UG-test 完整的数据集上进行. 根据组 委会提供的结果，本文方法训练的模型最终在 UG-test 上测试的 $\mathrm{mAP}$ 为 $49.22 \%$. 从表 7 可以看出,

(1) http://cvpr2020.ug2challenge.org/program19/leaderboard19 t2.html 
HRI-DET 队伍提出的方法效果最好. 但是该队伍 使用了超过 10000 幅图像进行训练, 而本文方法 仅使用 4000 多幅图像训练就能达到仅次于它的效 果. KODNet 在 UG-test 上的部分测试结果如图 12 所示.

表 6 KODNet 在 UG-test 的评估结果

\begin{tabular}{|c|c|c|c|c|c|}
\hline 方法 & 针框设计 & 数据清洗 & 数据增强 & 后处理 & $\mathrm{mAP} / \%$ \\
\hline 基准 & & & & & 28.63 \\
\hline KODNet & $\sqrt{ }$ & & & & 30.86 \\
\hline KODNet & $\sqrt{ }$ & $\sqrt{ }$ & & & 42.17 \\
\hline KODNet & $\sqrt{ }$ & $\sqrt{ }$ & $\sqrt{ }$ & $\sqrt{ }$ & 49.60 \\
\hline KODNet & $\sqrt{ }$ & $\sqrt{ }$ & $\sqrt{ }$ & $\sqrt{ }$ & 54.79 \\
\hline
\end{tabular}

表 7 KODNet 与其他方法在 UG-test 的 mAP \%

\begin{tabular}{ll}
\hline \multicolumn{1}{c}{ 方法 } & mAP \\
\hline CAS-Newcastle & 20.46 \\
CAS_NEU & 25.09 \\
Mt.Star & 31.24 \\
本文 & 49.22 \\
HRI-DET & 52.71 \\
\hline
\end{tabular}

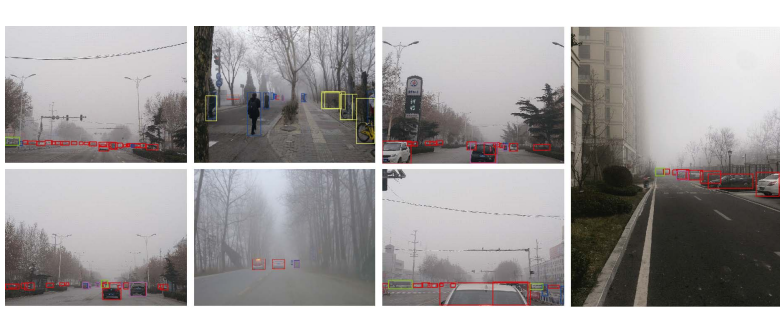

自行车; 一摩托车; 一人; 一公共汽车; 一汽车

图 12 KODNet 在 UG-test 的部分测试结果

\section{5 结 语}

本文梳理了真实有雾场景下目标检测的方法 和难点，并探讨了促进该场景下目标检测研究的 方向，即数据集的构建与处理和不同有雾场景下 目标检测框架的提出. 首先, 设计了一种系统化的、 具有真实感的有雾图像合成方法, 将 MS COCO 图 像通过一种低成本的方式构建成合成有雾场景下 的目标检测数据集. 同时, 对真实有雾场景下的目 标检测数据集进行数据清洗、数据增强和后处理等 操作，并验证了数据集处理可以有效地提升该场 景下目标检测的性能, 为今后研究有雾场景下的 目标检测提供一个解决思路. 其次，探讨了 2 个能 够促进有雾场景下目标检测性能的方向，基于此 提出 KODNet 和 DONet. KODNet 将统计先验知识
用于目标检测网络的针框参数设置, 使其引导通 用目标检测模型更好地适应特殊的目标检测场景, 但是使用目标分布这一先验知识进行引导的目标 检测框架在性能提升上有明显的上限. DONet 将去 雾模型和目标检测模型进行联合优化学习, 并通 过大量的实验证明了该框架可以有效提高真实有 雾场景下目标检测的性能. 由于联合优化的输人 要适应去雾模型和检测模型, 所以输人图像并未 做出任何处理，该方面的限制也影响着真实有雾 场景下的目标检测性能.

在未来工作中, 将从知识引导这一方向进一 步考虑影响有雾场景下目标检测性能的域偏移问 题, 并将知识引导和联合优化这 2 个工作结合起 来，进一步扩充当前真实有雾场景下目标检测的 解决方法.

\section{参考文献(References):}

[1] Li B Y, Peng X L, Wang Z Y, et al. AOD-Net: all-in-one dehazing network[C]//Proceedings of the IEEE International Conference on Computer Vision. Los Alamitos: IEEE Computer Society Press, 2017: 4780-4788

[2] Ren S Q, He K M, Girshick R, et al. Faster R-CNN: towards real-time object detection with region proposal networks[J]. IEEE Transactions on Pattern Analysis and Machine Intelligence, 2017, 39(6): 1137-1149

[3] Li B Y, Ren W Q, Fu D P, et al. Benchmarking single image dehazing and beyond[J]. IEEE Transactions on Image Processing, 2019, 28(1): 492-505

[4] Liu Y, Zhao G L, Gong B Y, et al. Improved techniques for learning to dehaze and beyond: a collective study[OL]. [2020-08-05]. https://arxiv.org/abs/1807.00202

[5] Yang W H, Yuan Y, Ren W Q, et al. Advancing image understanding in poor visibility environments: a collective benchmark study[J]. IEEE Transactions on Image Processing, 2020, 29: $5737-5752$

[6] Everingham M, Eslami S M A, van Gool L, et al. The pascal visual object classes challenge: a retrospective[J]. International Journal of Computer Vision, 2015, 111(1): 98-136

[7] Lin T Y, Maire M, Belongie S, et al. Microsoft COCO: common objects in context[C] //Proceedings of European Conference on Computer Vision. Heidelberg: Springer, 2014: 740-755

[8] Cai B L, Xu X M, Jia K, et al. DehazeNet: an end-to-end system for single image haze removal[J]. IEEE Transactions on Image Processing, 2016, 25(11): 5187-5198

[9] Mei K F, Jiang A W, Li J C, et al. Progressive feature fusion network for realistic image dehazing[C] //Proceedings of Asian Conference on Computer Vision. Heidelberg: Springer, 2019: 203-215

[10] He K M, Sun J, Tang X O. Guided image filtering[J]. IEEE Transactions on Pattern Analysis and Machine Intelligence, 2013, 35(6): 1397-1409 
[11] Nada H, Sindagi V A, Zhang H, et al. Pushing the limits of unconstrained face detection: a challenge dataset and baseline results[C] //Proceedings of the 9th International Conference on Biometrics Theory, Applications and Systems. Los Alamitos: IEEE Computer Society Press, 2018: Article No.8698561

[12] Sermanet P, Eigen D, Zhang X, et al. Overfeat: integrated recognition, localization and detection using convolutional networks[OL]. [2020-08-05]. https://arxiv.org/pdf/1312.6229

[13] Redmon J, Divvala S, Girshick R, et al. You only look once: unified, real-time object detection[C] //Proceedings of the 29th IEEE Conference on Computer Vision and Pattern Recognition. Los Alamitos: IEEE Computer Society Press, 2016: 779-788

[14] Redmon J, Farhadi A. YOLO9000: better, faster, stronger[C] //Proceedings of the 30th IEEE Conference on Computer Vision and Pattern Recognition. Los Alamitos: IEEE Computer Society Press, 2017: 7263-7271

[15] Redmon J, Farhadi A. YOLOv3: an incremental improvement[OL]. [2020-08-05]. https://arxiv.org/abs/1804.02767

[16] Liu W, Anguelov D, Erhan D, et al. SSD: single shot multibox detector[C] //Proceedings of the 14th European Conference on Computer Vision. Heidelberg: Springer, 2016: 21-37

[17] Lin T Y, Goyal P, Girshick R, et al. Focal loss for dense object detection[J]. IEEE Transactions on Pattern Analysis and Machine Intelligence, 2020, 42(2): 318-327

[18] Zhang S F, Wen L Y, Bian X, et al. Single-shot refinement neural network for object detection[C] //Proceedings of the IEEE Conference on Computer Vision and Pattern Recognition. Los Alamitos: IEEE Computer Society Press, 2017: 4203-4212

[19] Tian Z, Shen C H, Chen H, et al. FCOS: fully convolutional one-stage object detection[C] //Proceedings of the IEEE International Conference on Computer Vision. Los Alamitos: IEEE Computer Society Press, 2019: 9626-9635

[20] Girshick R, Donahue J, Darrell T, et al. Rich feature hierarchies for accurate object detection and semantic segmentation[C] //Proceedings of the IEEE Conference on Computer Vision and Pattern Recognition. Los Alamitos: IEEE Computer Society Press, 2014: 580-587

[21] He K M, Zhang X Y, Ren S Q, et al. Spatial pyramid pooling in deep convolutional networks for visual recognition[C] //Proceedings of European Conference on Computer Vision. Heidelberg: Springer, 2014: 346-361

[22] Girshick R. Fast R-CNN[C] //Proceedings of the IEEE International Conference on Computer Vision. Los Alamitos: IEEE Computer Society Press, 2015: 1440-1448

[23] Dai J F, Li Y, He K M, et al. R-FCN: object detection via region-based fully convolutional networks[C] //Proceedings of Advances in Neural Information Processing Systems. Cam- bridge: MIT Press, 2016: 379-387

[24] He K M, Gkioxari G, Dollár P, et al. Mask R-CNN[J]. IEEE Transactions on Pattern Analysis and Machine Intelligence, 2020, 42(2): 386-397

[25] Li Z M, Peng C, Yu G, et al. Light-Head R-CNN: in defense of two-stage object detector[OL]. [2020-08-05]. https://arxiv.org/ abs/1711.07264

[26] Cai Z W, Vasconcelos N. Cascade R-CNN: delving into high quality object detection[C] //Proceedings of the IEEE Conference on Computer Vision and Pattern Recognition. Los Alamitos: IEEE Computer Society Press, 2018: 6154-6162

[27] Li Y H, Chen Y T, Wang N Y, et al. Scale-aware trident networks for object detection[C] //Proceedings of the IEEE International Conference on Computer Vision. Los Alamitos: IEEE Computer Society Press, 2019: 6053-6062

[28] Godard C, Aodha O M, Firman M, et al. Digging into self-supervised monocular depth estimation[C] //Proceedings of the IEEE International Conference on Computer Vision. Los Alamitos: IEEE Computer Society Press, 2019: 3827-3837

[29] Ronneberger O, Fischer P, Brox T. U-Net: convolutional networks for biomedical image segmentation[C] //Proceedings of the 18th International Conference on Medical Image Computing and Computer-Assisted Intervention. Heidelberg: Springer, 2015: $234-241$

[30] Sakaridis C, Dai D X, van Gool L. Semantic foggy scene understanding with synthetic data[J]. International Journal of Computer Vision, 2018, 126: 973-992

[31] Chen K, Wang J Q, Pang J M, et al. MMDetection: open MMLab detection toolbox and benchmark[OL]. [2020-08-05]. https://arxiv.org/abs/1906.07155

[32] Xie S N, Girshick R, Dollár P, et al. Aggregated residual transformations for deep neural networks[C] //Proceedings of the IEEE Conference on Computer Vision and Pattern Recognition. Los Alamitos: IEEE Computer Society Press, 2017: 5987-5995

[33] Lin T Y, Dollár P, Girshick R, et al. Feature pyramid networks for object detection[C] //Proceedings of the 30th IEEE Conference on Computer Vision and Pattern Recognition. Los Alamitos: IEEE Computer Society Press, 2017: 936-944

[34] Huang Jingying. Research on haze-prior induced deep dehazing model[D]. Xiamen: Xiamen University, 2020(in Chinese) (黄静颖. 雾先验诱导的图像去雾深度学习方法研究[D]. 厦 门: 厦门大学, 2020)

[35] He K M, Zhang X Y, Ren S Q, et al. Deep residual learning for image recognition[C] //Proceedings of the 29th IEEE Conference on Computer Vision and Pattern Recognition. Los Alamitos: IEEE Computer Society Press, 2016: 770-778 